\title{
Elimination of Oxygen Interference in the Electrochemical Detection of Monochloramine, using in-situ pH Control at Interdigitated Elec- trodes
}

\author{
Ian Seymour, Benjamin O’Sullivan, Pierre Lovera, Alan O’Riordan and James F. Rohan \\ Tyndall National Institute, Cork, Ireland. \\ E-mail: \\ KEYWORDS Chlorine sensing, Water quality monitoring, In situ pH control, Amperometric Sensor, Interdigitated array, \\ Hypochlorous acid
}

\begin{abstract}
Disinfection by chloramination of water systems is an alternative to chlorination that is frequently used in North America. In such a case, monochloramine is used as the primary source of chlorine for disinfection. Regular monitoring of the residual concentrations of this species is crucial to ensure adequate disinfection. An amperometric sensor for monochloramine would provide fast, reagent free analysis, however the presence of dissolved oxygen in water complicates sensor development. In this work, we have explored the use of in-situ $\mathrm{pH}$ control as a method of eliminating oxygen as an interferent by conversion of monochloramine to dichloramine. The electrochemical reduction of dichloramine occurs outside the oxygen reduction window and is therefore not affected by oxygen concentration. Potential sweep methods were used to investigate the conversion of monochloramine to dichloramine at $\mathrm{pH}$ 3. The $\mathrm{pH}$ control method was used to calibrate monochloramine concentrations between 1 and $10 \mathrm{ppm}$, with a detection limit of $0.03 \mathrm{ppm}$. Tests were carried out in high alkalinity samples, wherein it was found that the sensitivity of this method effectively remained unchanged. Monochloramine was also quantified in the presence of common interferents (copper, phosphate and iron) which had no significant impact on the analysis
\end{abstract}

\section{1 - Introduction}

Disinfection of water systems is crucial to ensure safety of potable water, and is typically done using hypochlorous acid (HOCl) as a disinfecting agent.[1, 2] The measurement of the residual chlorine is important as it can determine the progress of the disinfection process. However, it is also crucial to monitor the by-products of the disinfection process, one of which is monochloramine (MCA). MCA is formed when $\mathrm{HOCl}$ reacts with ammonia $\left(\mathrm{NH}_{3}\right)$, shown in equation 1.[3,4]

$$
\mathrm{HOCl}+\mathrm{NH}_{3} \rightarrow \mathrm{NH}_{2} \mathrm{Cl}+\mathrm{H}_{2} \mathrm{O} \quad \text { Equation } 1
$$

The formation of MCA is governed by the concentration of nitrogen relative to chlorine. This is defined as the chlorine to nitrogen ratio $\left(\mathrm{Cl}_{2}: \mathrm{N}\right)$. MCA is produced when the $\mathrm{Cl}_{2}: \mathrm{N}$ is between $3: 1$ and 5:1.[5, 6] For a chlorine disinfection system, formation of $\mathrm{MCA}$ can be undesirable as it results in the "free" chlorine being converted to a combined chlorine species. This results in a lower residual chlorine and therefore a less adequate disinfection. Accurate monitoring of MCA formation in water systems reduces the risk of improper disinfection process.

Some water system utility companies, particularly in North America are switching to MCA as their primary water disinfectant source.[7] While typically MCA is regarded as a weaker disinfectant, there are numerous benefits to its use in water systems. It has been shown that MCA hydrolyses at a much slower rate than hypochlorous acid, and is far more stable in UV light[8, 9]. This means that the chlorine residual is more persistent and thus a more widespread and longer lasting disinfection is achieved. It has also been found to better penetrate biofilms, resulting in superior biocidal activity[10]. Perhaps a more significant advantage, is that MCA results in less toxic by-products. Water that has been treated with hypochlorous acid can result in the formation of trihalomethanes (THM)[11, 12]. These THMs have been shown to have mutagenic, cytotoxic and genotoxic effects, so minimization of their prevalence is desirable[13]. MCA treated water has been shown to have significantly lower concentrations of resulting THMs.[14] Treatment with MCA does have its disadvantages, most typically the formation of dichloramine (DCA) and trichloramine (TCA), which form as chlorine content increases, or the sample $\mathrm{pH}$ becomes acidic. Both are less powerful disinfectants than MCA and can lead to unpleasant tastes and smells in the water system. More significantly, the subsequent breakdown of TCA can lead to the formation of nitrates and nitrites. High concentrations of these can lead to nitrification of the water system which is hazardous to aquatic life.[1517] Low concentrations result in poor disinfection, but they can also be an indicator of high concentrations of organic matter in the system.[18] Because of this, MCA levels need to be monitored in water systems. The typical concentration expected in a chloraminated system ranges from $0.6 \mathrm{mg} / \mathrm{L}$ to $5 \mathrm{mg} / \mathrm{L}[19]$, and requires routine analysis to ensure it stays within this range.

Many methods exist to determine MCA concentrations, such as; spectrophotometry, chemical titrations, gas chromatography, liquid chromatography and mass spectrometry [20-22]. Simple alternatives to lab based approaches is to use a commercially available test kit specific for MCA.[23] This is a colorimetric test kit wherein a reagent containing phenate is added to the MCA solution and reacts to form an indophenol, which is green in colour.[24] The issue with these methods, however, is that a skilled operator is required to 
do the analysis, and additional reagents are required to be added that are environmentally undesirable. They also require sampling of the water system by an operator to remove an adequate volume for measurement. This increases the time and the cost associated with carrying out a measurement. By contrast, electrochemical methods are portable, low cost and highly sensitive.[25-27] Quantification using an electrochemical method would simplify this analysis as no additional reagents are required, and the device can be deployed and accessed remotely. The electrochemical analysis of MCA is undertaken by measuring the current associated with the reduction of MCA to ammonium and chloride. This is a one-step, two electron reduction as shown in Equation : [20,28]

$$
\mathrm{NH}_{2} \mathrm{Cl}+2 \mathrm{H}^{+}+2 e^{-} \leftrightarrow \mathrm{Cl}^{-}+\mathrm{NH}_{4}^{+}
$$

Equation 2

A key innovation in this paper is the elimination of oxygen as an interfering species. One of the major issues with electrochemical quantification of MCA is that dissolved oxygen in the solution is an interferent.[20, 29, 30] Dissolved oxygen undergoes reduction in the same electrochemical window that is used for the analysis of MCA. The reduction of DCA however, occurs at potentials outside of the oxygen reduction window, the reaction for which is shown in equation 3.[28]

$$
\mathrm{NHCl}_{2}+3 \mathrm{H}^{+}+4 e^{-} \rightarrow \mathrm{NH}_{4}^{+}+2 \mathrm{Cl}^{-} \quad \text { Equation } 3
$$

Conversion of MCA to DCA requires a pH shift to acidic conditions, resulting in the formation of a protonated MCA species, which breaks down to form DCA as shown in equations 4 and 5. [31,32]

$$
\begin{array}{ccc}
\mathrm{NH}_{2} \mathrm{Cl}+\mathrm{H}^{+} \leftrightarrow \mathrm{NH}_{3} \mathrm{Cl}^{+} & \text {Equation 4 } \\
\mathrm{NH}_{3} \mathrm{Cl}^{+}+\mathrm{NH}_{2} \mathrm{Cl} \leftrightarrow \mathrm{NHCl}_{2}+\mathrm{NH}_{4}^{+} & \text {Equation 5 }
\end{array}
$$

To convert MCA to DCA, in-situ $\mathrm{pH}$ control is required. In situ $\mathrm{pH}$ control has been shown by others using a ring disc electrode arrangement. Hydrolysis of water produced protons at an anode ring which diffused to the sensing disc electrode lowering the $\mathrm{pH}$ insitu, compared to the bulk solution, and enabled direct detection of metals.[33]. We have recently shown that an in-situ $\mathrm{pH}$ control method can facilitate the detection of hypochlorous acid by protonation of the hypochlorite ion.[34] Conversion to a single species removed the complexity involved with calibration. On-chip, the $\mathrm{pH}$ can be tailored to the required value by control of the applied current density.[35] The $\mathrm{pH}$ can be changed through the water splitting reactions of equations 6 and 7 wherein protons are produced as a result of oxygen gas evolution.[36]

$$
\begin{array}{cc}
\text { Cathode: } 2 \mathrm{H}^{+}+2 e^{-} \rightarrow \mathrm{H}_{2} & \text { Equation } 6 \\
\text { Anode: } 2 \mathrm{H}_{2} \mathrm{O} \rightarrow \mathrm{O}_{2}+4 \mathrm{H}^{+}+4 e^{-} & \text {Equation 7 }
\end{array}
$$

The electrochemical measurements in this work are performed on a generator-collector type device composed of two combs of interdigitated electrode arrays. A comb here refers to one half of the interdigitated electrode array. The working electrodes are spaced 2 $\mu \mathrm{m}$ apart while the counter electrode is $1.1 \mathrm{~mm}$ away from the sensors. By imposing an appropriate potential at one ("protonator") comb of electrodes, a $\mathrm{pH}$ change occurs in the local environment that tailors the $\mathrm{pH}$ at the other ("sensor") comb. That sensor can thus be used to perform sensing in $\mathrm{pH}$ conditions that differ from the bulk solution. It is vital that the counter electrode is relatively well removed spatially from the interdigitated combs. This ensures that the consumption of protons does not occur too close to the sensing electrode, which would inevitably prevent $\mathrm{pH}$ control. Using this approach, a local environment is created that is more acidic (or basic) than the bulk conditions. We apply this method to sensing of MCA by electrochemically shifting the $\mathrm{pH}$ at a sensor to more acidic conditions. Converting the MCA to DCA shifts the reaction of interest outside of the oxygen reduction voltage window thus removing oxygen as an interfering species, simplifying the analysis. The close spacing of the interdigitated electrodes ensures that $\mathrm{pH}$ control is established by the rapid diffusion of protons, so additional convection or fluidic forces are not required. Thus, this approach has the potential for in-line analysis deployment as required, for example, in water distribution systems. The schematic shown in Figure 1 shows how in-situ pH control creates a local acidic environment allowing for the sensor electrode to amperometrically detect DCA rather than MCA. 

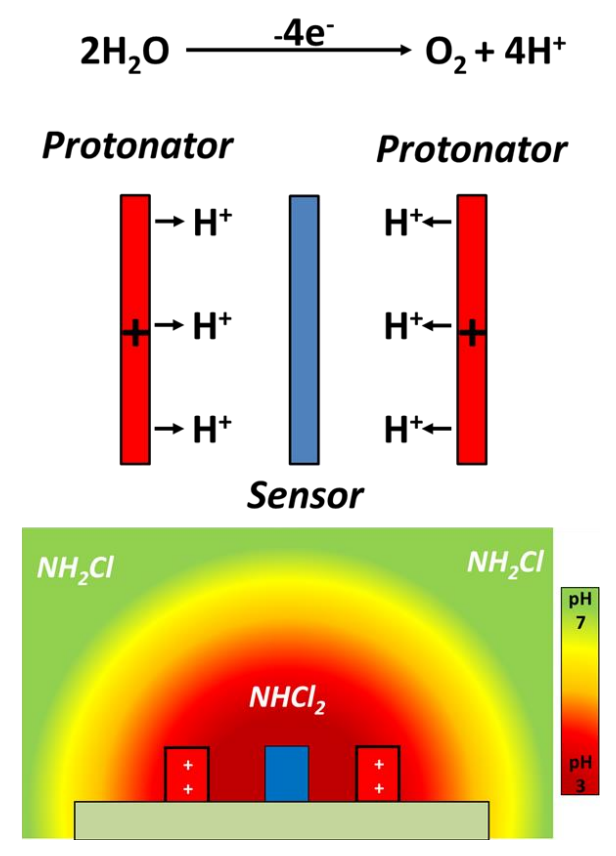

Figure 1 Schematic representation of the $\mathrm{pH}$ control method.

\section{2 - Experimental Section}

\section{1 - Electrode Fabrication:}

Silicon chip based devices were fabricated using methods similar to those described by Dawson et al.[37-39] Each chip consisted of two combs of gold working interdigitated electrodes, platinum pseudo reference and gold counter electrodes were also employed onchip. In brief, chips were designed to interface with external electronics via a microSD port to facilitate facile electrical connection. All of the devices were fabricated on 4-inch silicon wafers bearing a thermally grown $300 \mathrm{~nm}$ silicon dioxide layer. Blanket metal evaporations of Titanium $(10 \mathrm{~nm})$ and Gold $(100 \mathrm{~nm})$ using a Temescal FC-2000 E-beam evaporator and lift-off technique yields interdigitated microband $(55 \mu \mathrm{m} \times 1 \mu \mathrm{m} \times 60 \mathrm{~nm})$ structures with gaps between the combs of $2 \mu \mathrm{m}$. A second metal evaporation and lift-off process yields the interconnection tracks, contact pads and the gold counter electrode $(90 \mu \mathrm{m} \times 7 \mathrm{~mm})$. Finally, a third metal evaporation was performed to create the platinum pseudo reference electrode, however an external reference electrode was used in this work. To prevent unwanted interactions along the connection tracks, silicon nitride, which acts as an insulating layer was deposited by plasma enhanced chemical vapour deposition. Photolithography and dry etching were utilised to selectively open windows $(45 \mu \mathrm{m} \times 100 \mu \mathrm{m})$ in the insulating SiN layer over the microband electrodes for electrolyte access. Openings were also created over the counter and pseudo-reference electrodes and the contact pads. Each device contains six interdigitated electrode (sensors) which are separated by $0.94 \mathrm{~mm}$. Once the sensor fabrication is completed, a wafer was diced into 28 separate chip devices.

A custom-made holder cell was fabricated to allow measurement in small electrolyte volumes $(\approx 50 \mu \mathrm{L}$ to $5 \mathrm{mLs}$.). The cell was constructed from an aluminium base and a Teflon lid. Spring loaded probes (Coda Systems Ltd. PM4J Plain Radius Microprobes) were inserted into the lid in position above the peripheral contact pads, to permit electrical connection to external potientiostats. The cell was assembled with a Viton O-ring embedded in the lid to forma seal around the on-chip electrodes. Viton O-rings were chosen for their chemical resistance. The inner diameter of the O-ring was $7 \mathrm{~mm}$ with a cross section of $1.6 \mathrm{~mm}$ to allow an opening large enough to expose all six sensors, counter, and reference electrodes on the device to the electrolyte. Typical volumes measured were $5 \mathrm{~mL}$

\section{2 - Electrode Characterisation:}

Each chip was inspected using optical microscopy to identify any obvious defects or faults. Prior to any electrochemical characterisation chips were cleaned by immersion in acetone, iso-propyl alcohol and finally de-ionized water, each for a period of ten minutes. The chips were dried in a flow of nitrogen and placed in the chip holder. Electrochemical analysis was performed using an Autolab Bipotentiostat (MAC80150 with BA Module, Metrohm). Cyclic voltammograms (CV) were performed from $0 \mathrm{~V}$ to $0.6 \mathrm{~V}$ at $50 \mathrm{mV} / \mathrm{s}$ in $1 \mathrm{mM}$ ferrocene carboxylic acid (FCA, Sigma Aldrich, 97\%) dissolved in $10 \mathrm{mM}$ phosphate buffered saline (PBS, Sigma Aldrich). During these scans, the second interdigitated comb of electrodes was held at $0 \mathrm{~V}$. All electrochemical measurements were recorded versus a SCE, in solutions at room temperature $\left(21^{\circ} \mathrm{C}\right)$.

\section{3 - Platinum Plating on Protonator Electrode}

Platinum plating was carried out on one comb of the IDE array to enhance protonator performance. This was achieved by biasing the comb at $-0.5 \mathrm{~V}$ vs. SCE in a commercial Platinum DNS bath (Johnson Matthey). Plating times from 6 to 12 seconds were investigated 
but ultimately 8 second depositions were used. Scanning electron microscopy (SEM) and energy dispersive x-ray spectroscopy (EDX) were also used to characterise the platinum deposition.

\section{4 - Measurement of MCA by the Colorimetric Test}

Colorimetric measurement of MCA concentration was performed on the stock solution, and subsequent diluted working solutions to determine the concentration. This was undertaken as MCA can degrade over time, but more significantly the hypochlorous acid content of bleach also can drop over time. The stock therefore may not always be $200 \mathrm{ppm}$ depending on when the bleach was purchased. A commercial test kit was employed to determine the concentrations of the MCA solutions. The Hach colorimeter and indophenol method was used for this quantification. $10 \mathrm{~mL}$. of the MCA stock solution was added to a clean sample vial. This was put into the colorimeter and a blank measurement was taken. Monochlor F reagent was then added to the sample vial. This reagent was purchased as a pre-weighed sachet of powder, the entire contents of which were added to the sample. As the reagent reacted with the sample, the solution gradually became green with a maximum colouration achieved after a 5-minute reaction time. This was then put back into the colorimeter and a measurement was taken. This method is only sensitive to concentrations between 0.5 ppm and 5 ppm MCA, so dilutions were required to determine the concentration of stock solutions.

\section{5 - Electrochemical Analysis of Acidified MCA Solutions}

Solutions of MCA in artificial drinking water (ADW) were acidified to $\mathrm{pH} 3$ to determine the electrochemical behaviour of DCA on the IDEs. ADW was prepared by dissolving $1 \mathrm{~g}$ of sodium bicarbonate, $0.0654 \mathrm{~g}$ of magnesium sulphate (Sigma Aldrich, $99.5 \%$ anhydrous), $0.3414 \mathrm{~g}$ calcium sulphate dehydrate (Honeywell, 99\%), $0.007 \mathrm{~g}$ potassium phosphate dibasic (Fluka, $98 \%$ ), potassium phosphate monobasic (Sigma Aldrich, 99\%) and $0.01 \mathrm{~g}$ sodium nitrate (Sigma Aldrich, 99\%) in 10 L of deionised water. Sulphuric Acid $\left(0.1 \mathrm{M} \mathrm{H}_{2} \mathrm{SO}_{4}\right)$ was used to reduce the solution $\mathrm{pH}$ to the required value. The $\mathrm{pH}$ of each solution was confirmed with a $\mathrm{pH}$ meter (Hach). Electrochemical analysis of the acidified samples was performed using an Autolab potentiostat (MUX 101 with BA module) in a Faraday cage. The working electrode used was an IDE array with $1 \mu \mathrm{m}$ wide electrodes separated by $2 \mu \mathrm{m}$ gaps. The counter was an on-chip platinum electrode and the reference was a SCE. CVs were performed from $1.2 \mathrm{~V}$ to $0.2 \mathrm{~V}$ at $50 \mathrm{mV} / \mathrm{s}$. Scans were also performed at intermediate $\mathrm{pH}$ values to determine the $\mathrm{pH}$ dependence of DCA formation.

\section{6 - pH Control in MCA Solutions at $\mu$ IDE Arrays}

Electrochemical analysis of MCA solutions via $\mathrm{pH}$ control was carried out using an Autolab bipotentiostat in a Faraday cage. The same electrochemical parameters were used as in section 0, initially. The starting potential was reduced to $0.95 \mathrm{~V}$ for later work. The protonator electrode was held at $1.57 \mathrm{~V}$ or $1.65 \mathrm{~V}$ depending on the matrix conditions. Working samples were made by diluting the MCA stock solution with ADW. Typical concentrations of 0.5 to 5 ppm MCA were used, with 10 ppm samples used to study the upper detection limit.

\section{7 - Effects of Interferents and Matrix Composition on MCA Detection}

Solutions of MCA with high alkalinity were prepared by the addition of sodium bicarbonate $(0.84 \mathrm{~g} / 500 \mathrm{ml}$ ADW stock). Different MCA sample solutions were spiked with 1 ppm each of iron, copper and phosphate (as iron (II) chloride, copper sulphate and sodium phosphate monobasic) to determine their interferents effect on the MCA analysis.

\section{3 - Results and Discussion}

\section{1 - Device Characterisation:}

Devices were fabricated with an inter-electrode comb spacing of $2 \mu \mathrm{m}$. Each comb of interdigitated electrodes can be addressed separately allowing for generator-collector type sensing applications. In this work, platinum was plated onto one comb of the interdigitated array which was to be used as the protonator electrode. The platinum deposition was performed by biasing the one comb of the gold interdigitated array at $-0.5 \mathrm{~V}$ for 8 seconds. Figure 2 (A) is a SEM image showing that platinum was successfully deposited solely onto one comb of electrodes, with the other comb being unaffected. The SEM indicates that approximately a $100 \mathrm{~nm}$ thick deposit was achieved. The EDX shown in Figure 2 (B) further shows the presence of platinum on the comb of electrodes, indicted by the platinum peaks highlighted with the grey arrows. Gold was also detected, as only a thin layer of platinum was deposited, through which the X-rays penetrated detecting the underlying gold. Following SEM and EDX, the sensors were electrochemically characterized using FCA. Figure 2 (C) shows a typical scan performed in single mode (i.e. no bias at the collect comb of electrodes) at the gold comb and the platinum comb. The combs were swept from $0 \mathrm{~V}$ to $0.6 \mathrm{~V}$ at $50 \mathrm{mV} / \mathrm{s}$, oxidising the FCA to FCA ${ }^{+}$. The $\mathrm{CV}_{\mathrm{s}}$ showed different behaviours for the gold and platinum combs of electrodes, further indicating successful deposition of platinum. The reduction event seen between $0 \mathrm{~V}$ and $0.1 \mathrm{~V}$ for platinum is the onset of oxygen reduction, as platinum shows better catalytic activity towards this reaction than gold. In both cases, the CVs showed a peak associated with FCA oxidation at approximately $0.35 \mathrm{~V}$. Steady state behaviours were expected for the ultramicron scale electrodes, however diffusional overlap resulted in the array behaving as 
one large electrode, as opposed to multiple smaller electrodes. The platinum comb of electrodes showed a higher current for the FCA oxidation due to the increased surface as a result of the plating procedure.

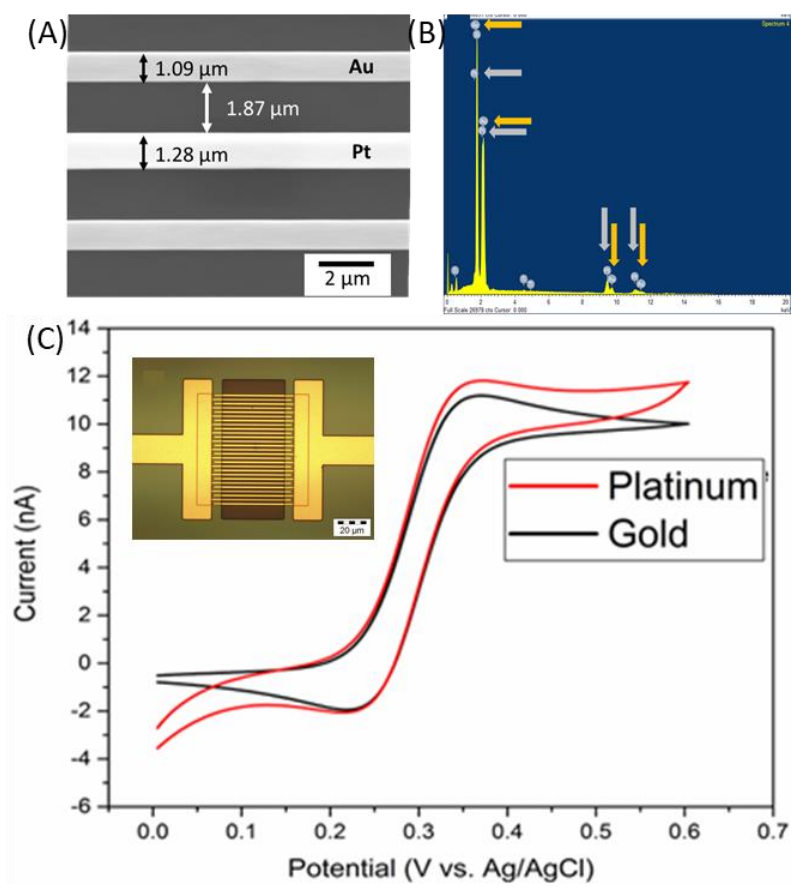

Figure 2 (A) SEM image of the IDE array showing one comb plated with platinum. (B) EDX spectra of the plated platinum comb of electrod es showing both platinum and gold peaks. (C) CV of a gold comb (black) and a platinum comb (red) of electrodes in a solution of $1 \mathrm{mmol} / \mathrm{L}$ FCA in $10 \mathrm{mmol} / \mathrm{L}$ PBS. CV's were performed from $0 \mathrm{~V}$ to $0.6 \mathrm{~V}$ at $50 \mathrm{mV} / \mathrm{s}$. The inset shows an image of the IDE taken at $100 \times$ magnification.

Following characterisation in single mode, the devices were characterised in generator-collector mode (i.e. a bias imposed on the collector). In this case, the generator comb of electrodes was swept from $0 \mathrm{~V}$ to $0.6 \mathrm{~V}$ at $50 \mathrm{mV} / \mathrm{s}$, while the collector was biased at 0 $\mathrm{V}$. The generator comb oxidised the FCA to FCA+. The FCA+ species diffused across the gap to the collector electrode, which subsequently reduced it back to FCA. This is a phenomenon known as redox cycling and can be used to boost signals as described by Wahl et al.[40] The voltammograms shown in Figure 3 were typical of a fully working array. Figure 3 shows a comparison between a gold-gold IDE, and a gold-platinum IDE. In both cases, gold was used as the generator electrode with the collector being either platinum or gold. It was found that steady-state behaviour was achieved as the bias at the collector electrodes prevented diffusional overlap. No significant change in behaviour was observed between the gold-gold IDE and the gold-platinum IDE other than an increased collection efficiency. The collection efficiency is a measure of how much species generated at the generator is detected by the collector, and is calculated by expressing the collector current as a percentage of the generator current. A collection efficiency of 91.1\% was calculated for the gold-gold array, which indicated that a significant portion of the generated $\mathrm{FCA}^{+}$was observed at the collector. The collection efficiency of the gold-platinum IDE however, was calculated to be $95.7 \%$, an increase of $4.6 \%$ over the gold-gold array. The reason for the increase in collection efficiency resulted from increasing the width of the electrode, which subsequently decreased the gap between the generator and the collector electrodes. The inset shown in Figure 3 shows the current measured at gold and platinum electrodes when a potential where oxygen evolution occurred was applied to them in an ADW solution. Both electrodes were held at the same potential and it was found that the current measured at platinum was significantly higher than gold over multiple scans. As the $\mathrm{pH}$ control method requires oxygen evolution to produce hydrogen ions, the higher current measured at platinum indicates that it is a superior protonator material. For this reason, it was chosen as the protonator material in each of the $\mathrm{pH}$ control experiments. 


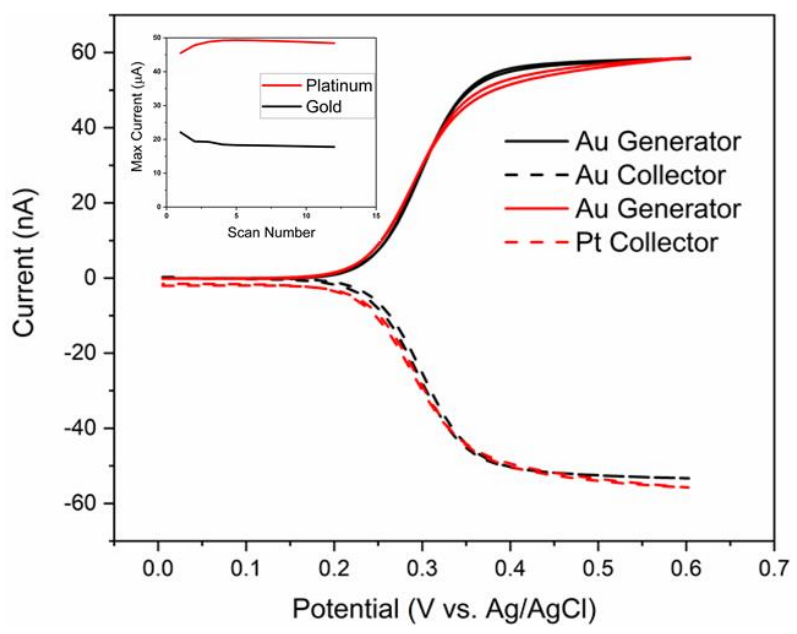

Figure $3 \mathrm{CVs}$ of a gold-gold array (black) and a gold-platinum array (red) in generator collector mode. CVs were performed at the generator (solid line) in 1 mmol/L FCA in 10 $\mathrm{mmol} / \mathrm{L}$ PBS from $0 \mathrm{~V}$ to $0.6 \mathrm{~V}$ at $50 \mathrm{mV} / \mathrm{s}$ while the collector (dashed line) was biased at $0 \mathrm{~V}$. The inset shows the comparison of platinum (red) and gold (black) performance as a protonator over multiple scans.

\section{2 - pH Dependence of DCA Formation:}

To determine the $\mathrm{pH}$ value at which DCA is dominant and detectable by amperometry, LSVs were performed in samples with 2.5 ppm MCA in ADW that was adjusted to various $\mathrm{pH}$ values. This was performed by making up samples of MCA in ADW at $\mathrm{pH} 8.5$ and subsequently acidifying each sample with $0.1 \mathrm{M} \mathrm{H}_{2} \mathrm{SO}_{4}$ until the desired $\mathrm{pH}$ was achieved. Figure 4 (A) shows the result of performing an LSV from $0.95 \mathrm{~V}$ to $0.2 \mathrm{~V}$ at $50 \mathrm{mV} / \mathrm{s}$ at each $\mathrm{pH}$ solution. It was found that at near neutral $\mathrm{pH}$ conditions, little to no DCA was detected. Full conversion to DCA was not observed until the sample $\mathrm{pH}$ was acidified to $\mathrm{pH} 3$, indicated by the steady state response. Subsequently, a calibration was performed in solutions of MCA acidified to $\mathrm{pH} 3$. Sample concentrations between 1 and 5 ppm MCA were used for this calibration as this range encompasses the average values expected when using MCA as a disinfectant. Each sample was acidified to $\mathrm{pH} 3$ to ensure the complete conversion of MCA to DCA. LSVs were performed in each solution from $1.2 \mathrm{~V}$ to $0.2 \mathrm{~V}$. These parameters were chosen as this allows a surface gold oxide to form. The reduction potential can be used as an indicator of sample $\mathrm{pH}$, which was a useful internal probe to determine if the $\mathrm{pH}$ control method is sufficient. Figure 4 (B) shows the LSVs in each concentration. A steady state response was observed for the reduction of DCA and the inset shows the corresponding calibration plot. A sensitivity to DCA of $0.485 \mathrm{nA} / \mathrm{ppm}$ was achieved with an $\mathrm{R}^{2}$ of 0.999 , showing that the reduction current was directly related to the MCA concentration. 

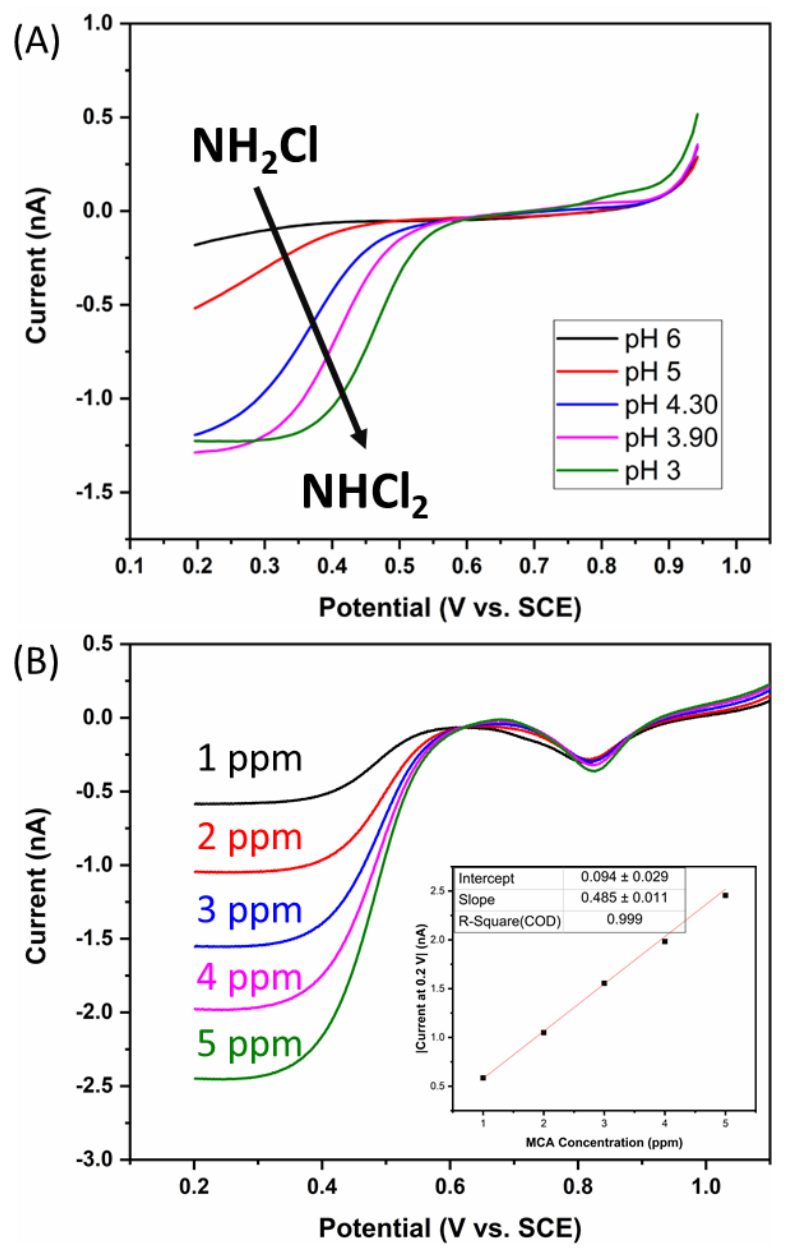

Figure 4 (A) The dependence of sample $\mathrm{pH}$ on the formation of DCA. (B) Electrochemical reduction of DCA at the gold comb of an IDE. CVs were performed in various concentrations of MCA in ADW that were subsequently adjusted to $\mathrm{pH}$ 3. LSVs were performed from $1.2 \mathrm{~V}$ to $0.2 \mathrm{~V}$ at $50 \mathrm{mV} / \mathrm{s}$. The inset shows the calibration plot with a slope of 0.485 and an $R^{2}$ of 0.999 , Error bars of triplicate measurements are within the data points

\section{3 - Analysis of MCA Samples by Conversion to DCA using in-situ pH Control:}

As it was determined that the samples were converted to DCA at $\mathrm{pH} 3$, which was quantifiable with the developed sensors, tests were performed using the proposed $\mathrm{pH}$ control method. Figure 5 (A) shows the initial LSVs performed in a $2 \mathrm{ppm}$ sample of MCA. The voltammogram shows a comparison between the situation when $\mathrm{pH}$ control was "off" and subsequently when $\mathrm{pH}$ control was "on". The LSVs involved scanning the sensor comb of electrodes from $1.2 \mathrm{~V}$ to $0.2 \mathrm{~V}$ at $50 \mathrm{mV} / \mathrm{s}$ while the protonator combs was unbiased ( $\mathrm{pH}$ control off) or biased at $1.57 \mathrm{~V}$ (pH control on). When the $\mathrm{pH}$ control technique was not applied, the solution was completely MCA, therefore now activity is seen in this potential window. The gold oxide reduction peak is seen at $0.3 \mathrm{~V}$, which was indicative of the $\mathrm{pH} 8.5$ solution. When the $\mathrm{pH}$ control method was applied, the local solution becomes $\mathrm{pH} 3$ and therefore the MCA was converted to DCA. In this case, a reduction event was observed which was similar to the equivalent scan performed in the chemically adjusted scans shown in Figure 4 (B). The gold oxide reduction peak is shifted to a potential of $0.75 \mathrm{~V}$ indicating a more acidic environment than the initial conditions. Figure 5 (B) shows a comparison of LSVs performed in samples saturated with oxygen and samples wherein the oxygen had been purged entirely by nitrogen degassing. It was clear that oxygen had no impact on the amperometric detection of DCA, so oxygen interference had been eliminated. The inset shows a scan performed using a commercial microdisc electrode in a $50 \mathrm{ppm}$ solution of MCA. In this case, the electrode was swept from $0.4 \mathrm{~V}$ to $-0.8 \mathrm{~V}$ at $50 \mathrm{mV} / \mathrm{s}$, which is the window wherein we can observe MCA reduction. A comparison between saturated and purged samples is shown and it is clear that oxygen has a significant impact on MCA detection. It was found that a $0.6 \mu \mathrm{A}$ difference was attributed to the presence of oxygen, effectively doubling the signal related to the $50 \mathrm{ppm}$ sample. For a $5 \mathrm{ppm}$ sample, this would correspond to a near ten-fold increase in signal. Variability of oxygen concentration therefore creates significant difficulty in the detection of MCA. The pH control method was applied to various concentrations of MCA in ADW as shown in Figure 5 (C). In these LSVs, the sensor comb was swept from $0.95 \mathrm{~V}$ to $0.2 \mathrm{~V}$ at $50 \mathrm{mV} / \mathrm{s}$ while the protonator comb was biased at $1.57 \mathrm{~V}$. As the $\mathrm{pH}$ control parameters were established, the generation of the oxide was no longer necessary. It had also been found that the presence of chlorine was causing dissolution of gold at $1.2 \mathrm{~V}$, therefore the lifetime of the sensor was improved by narrowing the potential window. In each concentration, similar behaviour was observed for the $\mathrm{pH}$ control method as was initially observed in the chemically adjusted samples. The typical range for MCA is $1 \mathrm{ppm}$ to $5 \mathrm{ppm}$, however this sensor was tested up to $10 \mathrm{ppm}$ MCA. This was undertaken as MCA can be formed if an excess of 
hypochlorite is present. In such a case, extreme MCA concentrations may be observed. The sensor was tested in such conditions to determine if the high concentrations could be effectively converted to DCA. Figure 5 (D) shows the calibration plot for the scans shown in Figure 5 (C). Again, a good linearity was observed with an $\mathrm{R}^{2}$ value of 0.998 . The measured sensitivity was found to be $0.385 \mathrm{nA} / \mathrm{ppm}$, which was lower than the scans performed in the chemically adjusted samples. This has been attributed to the lack of gold oxide formation and subsequent reduction, which did not occur in these scans. The formation of gold oxide and then reduction ensured a reproducible electrode surface. This process can remove some adsorbed species that may influence the sensitivity. As this was not performed in the $\mathrm{pH}$ control measurements to preserve the electrode lifetime, a slightly lower sensitivity was measured. This was confirmed by performing scans with the $\mathrm{pH}$ control method and the formation of a gold oxide, wherein the calibration plot indicates a sensitivity comparable to the $\mathrm{pH}$ adjusted samples. This data is shown in the supplementary information (SF1). A limit of detection was calculated for this method using the standard error of the estimate approach (SEq1).[41] However, rather than using a blank sample, seven replicates of a sample with $0.5 \mathrm{ppm}$ MCA were used resulting in a calculated limit of detection of 0.03 ppm. From this calibration, a series of samples containing 2 ppm MCA were compared across 5 sensors. These concentrations of MCA present were calculated using the calibration plot and simultaneously by using the colorimetric method. With the colorimetric being used as the standard approach, this sensing approach showed an average variation of $3.08 \%$ with a maximum deviation of $4.62 \%$.
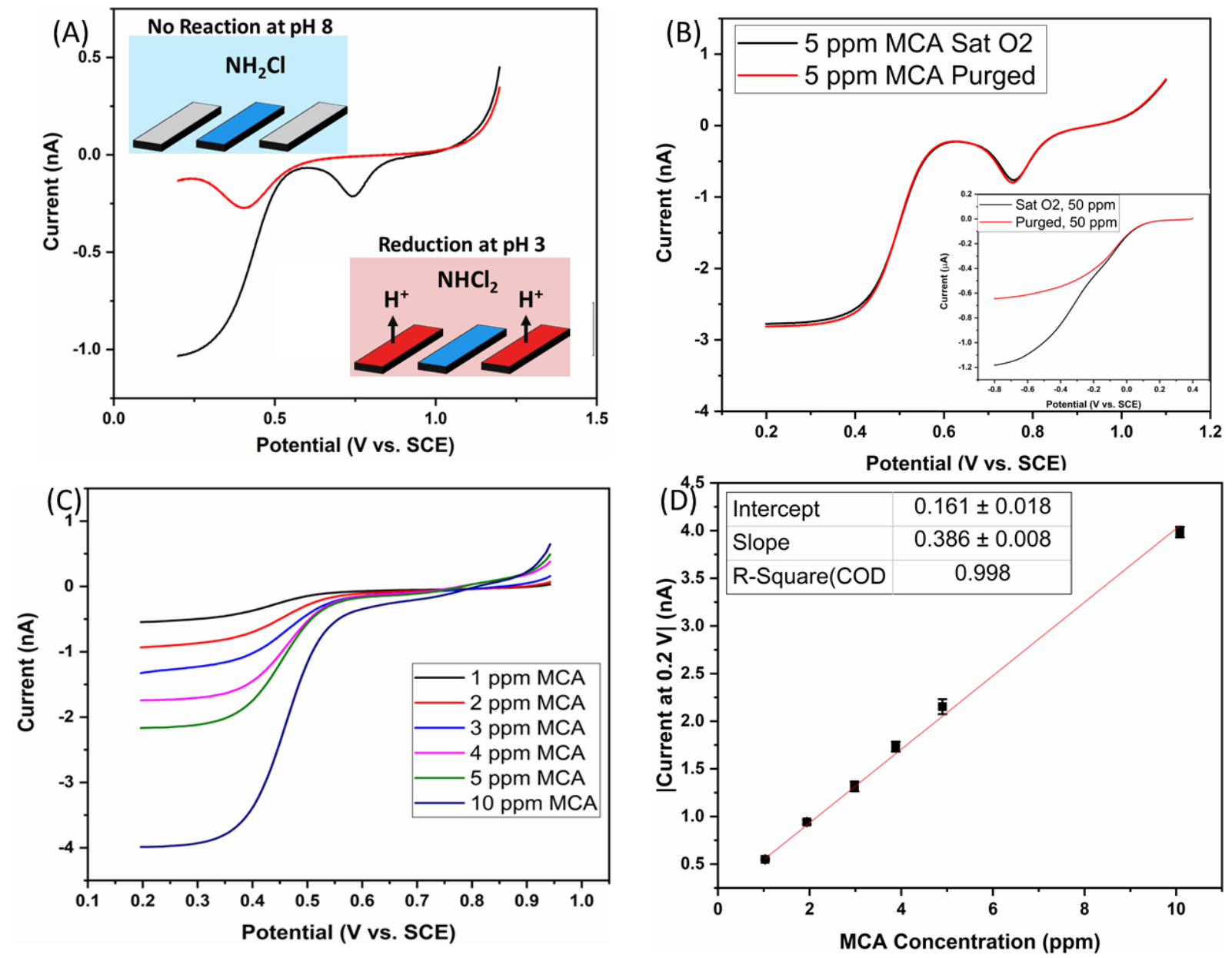

Figure 5 (A) Comparison of LSVs with $\mathrm{pH}$ control off (red) and on (black). CVs were performed at a gold comb of an IDE from $1.2 \mathrm{~V}$ to $0.2 \mathrm{~V}$ at $50 \mathrm{mV} / \mathrm{s}$ with the platinum protonator comb biased at $1.57 \mathrm{~V}$. The insets show a schematic of the electrode environment. (B) Comparison of LSVs in 5 ppm MCA samples with the pH control method applied at high an low concentrations of oxygen. The inset shows LSVs performed at a commercial microdisc electrode in 50 ppm sa mples of MCA at high and low oxygen concentrations from $0.4 \mathrm{~V}$ to $-0.8 \mathrm{~V}$ at $50 \mathrm{mV} / \mathrm{s}$. (C) LSVs in MCA samples from 1 to $10 \mathrm{ppm}$ with the applied pH control method. LSVs were performed at a gold comb of electrodes from $0.95 \mathrm{~V}$ to $0.2 \mathrm{~V}$ at $50 \mathrm{mV} / \mathrm{s}$ with the platinum protonator biased at $1.57 \mathrm{~V}$. (D) The calibration plot for the scans performed in (C).

\section{4 - Determination of the Effects of Matrix Composition and Common Interferents}

Oxygen is not the only anticipated interferent in water, as water systems can be quite complex. Therefore, tests were performed to determine the viability of the sensor in the presence of such interference. The sample alkalinity was of the most concern for this testing method as this can increase the buffering capacity of water. As the proposed method relies on changing $\mathrm{pH}$, high buffering capacity can add difficulty to this approach. The alkalinity of a water sample can be quite variable and typically is not a huge health concern, so high alkalinities can be common in some water systems. High alkalinity is expected to have approximately 500 ppm of carbonates or bicarbonates with 1000 ppm being very high. For this reason, tests were performed in samples of ADW with the addition 
of 1000 ppm sodium bicarbonate as a worst-case scenario. Figure 6 (A) shows the LSVs performed using the pH control method in high alkalinity samples. The major difference in parameters was an increase in the protonator potential from $1.57 \mathrm{~V}$ to $1.65 \mathrm{~V}$. This was required to get the desired $\mathrm{pH}$ control. The LSV performed at the sensor comb was kept at $0.95 \mathrm{~V}$ to $0.2 \mathrm{~V}$ at $50 \mathrm{mV} / \mathrm{s}$. The inset shows a calibration plot with an $\mathrm{R}^{2}$ of 0.998 , indicating a good linearity. Interestingly, the sensitivity measured was $0.363 \mathrm{nA} / \mathrm{ppm}$. This is only slightly lower that the sensitivity measured in the low alkalinity samples $(0.386 \mathrm{nA} / \mathrm{ppm})$ which indicates that the sensor performance is not significantly affected by the alkalinity. The limit of detection in this case was also measured to be 0.03 ppm using the same approach as for the low alkalinity samples. The increase to $1.65 \mathrm{~V}$ was employed as the measured current at the protonator was lower in the high alkalinity samples. If the protonator was biased with a current rather than a potential, this potential change would not be required. However due to the limitations of the potentiostat, a current could not be applied at one comb of electrodes while a potential sweep was performed at the other without the presence of a second counter electrode. As with the low alkalinity samples, a series of $2 \mathrm{ppm}$ samples were made in the high alkalinity ADW and tested across 5 sensors. In this case an average variability of $2.47 \%$ was observed with a maximum variation of $6.3 \%$. Figure 6 (B) shows the result of a series of LSVs performed in a high alkalinity sample of $2.5 \mathrm{ppm}$ MCA in which $1 \mathrm{ppm}$ of each interferent was added. The concentrations of interferent added represent again a worst-case scenario for each species. This concentration represents a typical upper level for copper and a significantly high level for phosphate. For iron higher concentrations may be found, however at 1 ppm iron is observable without additional equipment so its presence is obvious. No obvious events were observed for each species, as shown in the supplementary information (SF2), an increased current was measured in the presence of each species. A $4.6 \%$ increase over the expected current for a 2.5 ppm MCA sample was observed for iron. For phosphate and copper the increase was found to be $3.5 \%$ and $2.4 \%$ respectively.
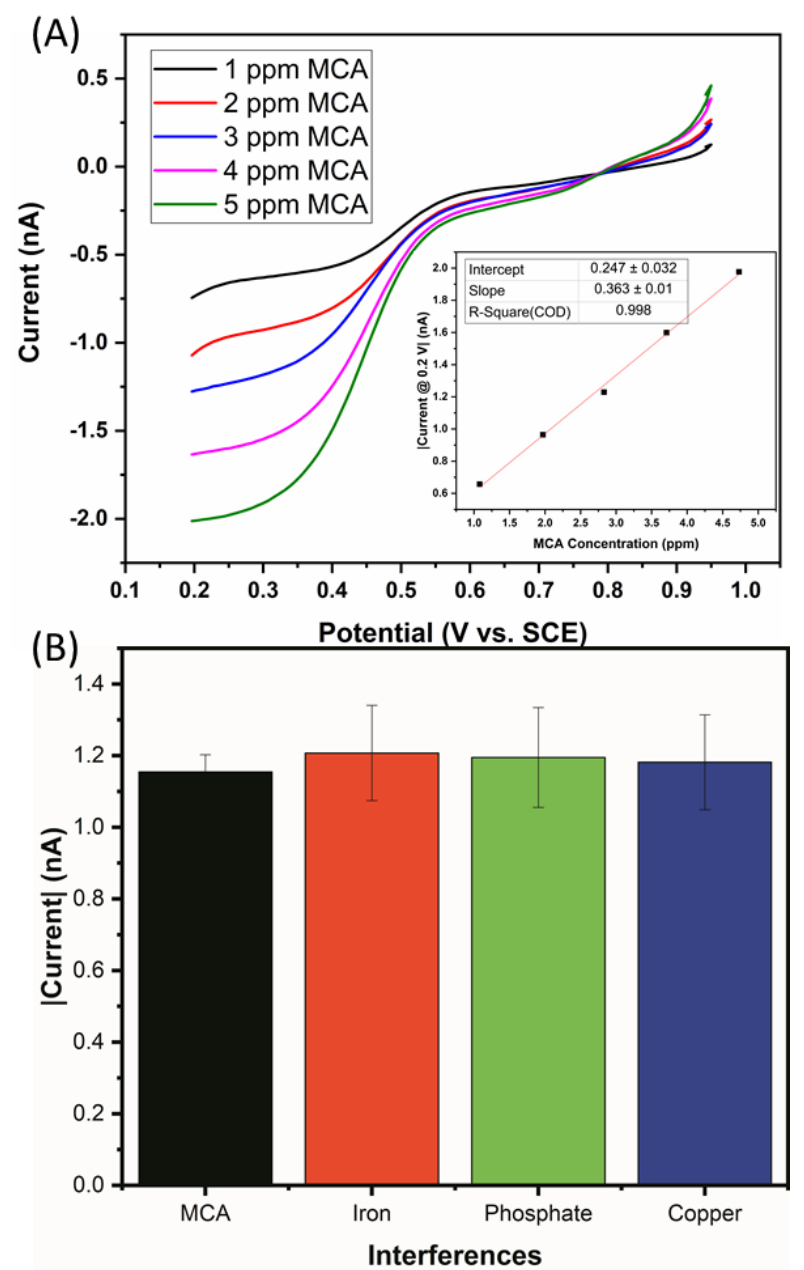

Figure 6 (A) LSVs in various concentrations of MCA in high alkalinity ADW. LSVs were performed at a gold comb of electrodes from $0.95 \mathrm{~V}$ to $0.2 \mathrm{~V}$ at $50 \mathrm{mV} / \mathrm{s}$ with the platinum protonator biased at $1.65 \mathrm{~V}$. The inset shows the calibration plot with a slop of 0.363 and an $\mathrm{R}^{2}$ of 0.998 . (B) Comparison of a 2.5 ppm MCA sample to equivalent samples spiked with $1 \mathrm{ppm}$ of Iron, Phosphate and Copper 


\section{Conclusions}

In this paper, we have demonstrated that reliable, reagent-free detection of MCA was achieved by using a pH control method. This method eliminates oxygen as an interfering species, which is one of the key difficulties associated with amperometric detection of MCA. The developed sensor was calibrated between 0 and 5 ppm MCA, which is the typical range expected in potable water, showing both accuracy and precision in measurements, with deviations of less than $6 \%$ and a detection limit calculated at 0.03 ppm MCA. The upper extreme was tested using a 10 ppm MCA sample, which was quantified with no loss of sensor performance. This range of concentrations far surpass the anticipated limits of potable water, indicating that the developed sensor is more than suitable for real world applications. The greatest anticipated challenge facing the use of $\mathrm{pH}$ control in real water samples is the inherent buffering capacity of water due to its alkalinity. In this work, we have evaluated the detection method at a potential worst-case scenario for alkalinity wherein the concentration of carbonates was $1000 \mathrm{ppm}$ and found that sensor performance was not impacted. The presence of common interferents in water, showed little to no impact on the detection method and MCA was still quantifiable in the presence of such compounds.

\section{AUTHOR INFORMATION}

\section{Corresponding Author}

* E-mail: alan.oriordan@tyndall.ie

\section{Author Contributions}

All authors have given approval to the final version of the manuscript.

\section{ACKNOWLEDGMENT}

This publication has emanated in part from research supported by a research grant from Science Foundation Ireland and the Department of Agriculture, Food and Marine on behalf of the Government of Ireland under the Grant 16/RC/3835 (VistaMilk), and supported from research conducted with the financial support of Science Foundation Ireland (SFI) and is co-funded under the European Regional Development Fund under Grant Number 13/RC/2077 (Connect).

\section{REFERENCES}

[1] M. Szili, I. Kasik, V. Matejec, G. Nagy, B. Kovacs, Poly(luminol) based sensor array for determination of dissolved chlorine in water, Sensors and Actuators B: Chemical, 192(2014) 92-8.

[2] Y. Dong, G. Li, N. Zhou, R. Wang, Y. Chi, G. Chen, Graphene Quantum Dot as a Green and Facile Sensor for Free Chlorine in Drinking Water, Anal Chem, 84(2012) 8378-82.

[3] J.F.T. Berliner, THE CHEMISTRY OF CHLORAMINES, Journal of the American Water Works Association, 23(1931) $1320-33$.

[4] M.K. Silva, I.C. Tessaro, K. Wada, Study of the formation of stable high concentrated monochloramine solutions, 2nd Mercosur Congress on Chemical Engineering, (2005).

[5] R.L. Wolfe, N.R. Ward, B.H. Olson, Inorganic Chloramines as Drinking Water Disinfectants: A Review, Journal of the American Water Works Association, 76(1984) 74-88.

[6] AWWA, Water Chlorination and Chloramination Practices and Principles, 2nd Edition ed.: American Water Works Association; 2011.

[7] L.J. Rose, E.W. Rice, L. Hodges, A. Peterson, M.J. Arduino, Monochloramine Inactivation of Bacterial Select Agents, Applied and Environmental Microbiology, 73(2007) 3437-9.

[8] R.G. Rice, M. Gomez-Taylor, Occurrence of by-products of strong oxidants reacting with drinking water contaminants--scope of the problem, Environmental Health Perspectives, 69(1986) 31-44.

[9] G.C. White, Determination of Chlorine Residuals in Water and Wastewater Treatment, White's Handbook of Chlorination and Alternative Disinfectants2009, pp. 174-229.

[10] W.H. Lee, D.G. Wahman, P.L. Bishop, J.G. Pressman, Free Chlorine and Monochloramine Application to Nitrifying Biofilm: Comparison of Biofilm Penetration, Activity, and Viability, Environmental Science \& Technology, 45(2011) 1412-9.

[11] G.J. Kirmeyer, A.R. Foundation, Optimizing Chloramine Treatment: American Water Works Association; 2004.

[12] R.D. Morris, A.-M. Audet, I.F. Angelillo, T.C. Chalmers, F. Mosteller, Chlorination, chlorination by-products, and cancer: a meta-analysis, American journal of public health, 82(1992) 955-63.

[13] L. de Castro Medeiros, F.L.S. de Alencar, J.A. Navoni, A.L.C. de Araujo, V.S. do Amaral, Toxicological aspects of trihal omethanes: a systematic review, Environmental Science and Pollution Research, 26(2019) 5316-32.

[14] AWWA, Committee Report: Disinfection Survey, Part2-Alternatives, experiences, and future plans, Journal of the American Water Works Association, 100(2008) 110-24. 
[15] R.L. Wolfe, E.G.M. III, M.K. Davis, S.E. Barrett, Biological Nitrification in Covered Reservoirs Containing Chloraminated Water, Journal of the American Water Works Association, 8o(1988) 109-14.

[16] R.L. Wolfe, N.I. Lieu, G. Izaguirre, E.G. Means, Ammonia-oxidizing bacteria in a chloraminated distribution system: seasonal occurrence, distribution and disinfection resistance, Applied and Environmental Microbiology, 56(1990) 451-62.

[17] AWWA, Nitrification Prevention and Control in Drinking Water: American Water Works Association; 2013.

[18] P.J. Vikesland, K. Ozekin, R.L. Valentine, Effect of Natural Organic Matter on Monochloramine Decomposition: Pathway Elucidation through the Use of Mass and Redox Balances, Environmental Science \& Technology, 32(1998) 1409-16.

[19] WHO, Monochlorine in Drinking Water, Monochlorine in Drinking Water, WHO/SDE/WSH/o3.04/83 ed., World Health Organisatio n2oo4. [20] V.V. Rajasekharan, B.N. Clark, S. Boonsalee, J.A. Switzer, Electrochemistry of Free Chlorine and Monochloramine and its Relevance to the Presence of Pb in Drinking Water, Environmental Science \& Technology, 41(2007) 4252-7.

[21] B. Piela, P.K. Wrona, Electrochemical behavior of chloramines on the rotating platinum and gold electrodes, Journal of the Electrochemical Society, 150(2003) E255-E65.

[22] S. Kinani, B. Richard, Y. Souissi, S. Bouchonnet, Analysis of inorganic chloramines in water, TrAC Trends in Analytical Chemistry, 33(2012) 5567.

[23] Hach, DR3oo Pocket Colorimeter, Monochlor/Free Ammonia, Hach2020.

[24] D.L. Harp, Specific Determination of Inorganic Monochloramine in Chlorinated Wastewaters, Water Environment Research, 72(200o) 70613.

[25] A. Montrose, N. Creedon, R. Sayers, S. Barry, A. O'riordan, Novel single gold nanowire-based electrochemical immunosensor for rapid detection of bovine viral diarrhoea antibodies in serum, J Biosens Bioelectron, 6(2015) 1-7.

[26] N. Creedon, C. Robinson, E. Kennedy, A.O. Riordan, Agriculture 4.0: Development of Seriological on-Farm Immunosensor for Animal Health Applications, 2019 IEEE SENSORS, (2019) 1-4.

[27] D.W.M. Arrigan, Nanoelectrodes, nanoelectrode arrays and their applications, Analyst, 129(2004) 1157-65.

[28] P.K. Wrona, Electrode processes of chloramines in aqueous solutions1, Journal of Electroanalytical Chemistry, 453(1998) 197-204.

[29] W.H. Lee, J.G. Pressman, D.G. Wahman, P.L. Bishop, Characterization and application of a chlorine microelectrode for mea suring monochloramine within a biofilm, Sensors and Actuators B: Chemical, 145(2010) 734-42.

[30] W.H. Lee, D.G. Wahman, J.G. Pressman, Monochloramine-sensitive amperometric microelectrode: optimization of gold, platinum, and carbon fiber sensing materials for removal of dissolved oxygen interference, Ionics, (2015).

[31] P.P. Fehér, M. Purgel, A. Lengyel, A. Stirling, I. Fábián, The mechanism of monochloramine disproportionation under acidic conditions, Dalton Transactions, 48(2019) 16713-21.

[32] V.C. Hand, D.W. Margerum, Kinetics and mechanisms of the decomposition of dichloramine in aqueous solution, Inorganic Chemistry, $22(1983) 1449-56$

[33] T.L. Read, E. Bitziou, M.B. Joseph, J.V. Macpherson, In Situ Control of Local pH Using a Boron Doped Diamond Ring Disk Electrode: Optimizing Heavy Metal (Mercury) Detection, Anal Chem, 86(2014) 367-71.

[34] I. Seymour, B. O'Sullivan, P. Lovera, J. Rohan, A. O'Riordan, Electrochemical Detection of Free-Chlorine in Water Samples Facilitated by InSitu $\mathrm{pH}$ Control Using Interdigitated Microelectrodes, (2020).

[35] T.L. Read, M.B. Joseph, J.V. Macpherson, Manipulation and measurement of pH sensitive metal-ligand binding using electrochemical proton generation and metal detection, Chemical Communications, 52(2016) 1863-6.

[36] J. Rossmeisl, A. Logadottir, J.K. Nørskov, Electrolysis of water on (oxidized) metal surfaces, Chemical Physics, 319(2005) 178-84.

[37] K. Dawson, A. Wahl, R. Murphy, A. O’Riordan, Electroanalysis at Single Gold Nanowire Electrodes, The Journal of Physical Chemistry C, $116(2012)$ 14665-73.

[38] K. Dawson, A. Wahl, S. Barry, C. Barrett, N. Sassiat, A.J. Quinn, et al., Fully integrated on-chip nano-electrochemical devices for electroanalytical applications, Electrochimica Acta, 115(2014) 239-46.

[39] S. Barry, K. Dawson, E. Correa, R. Goodacre, A. O'Riordan, Highly sensitive detection of nitroaromatic explosives at discrete nanowire arrays, Faraday discussions, 164(2013) 283-93.

[40] A.J.C. Wahl, I.P. Seymour, M. Moore, P. Lovera, A. O'Riordan, J.F. Rohan, Diffusion profile simulations and enhanced iron sensing in generatorcollector mode at interdigitated nanowire electrode arrays, Electrochimica Acta, 277(2018) 235-43.

[41] B. Brunetti, About Estimating the Limit of Detection by the Signal to Noise Approach, Pharmaceutica Analytica Acta, o6(2015). 